\title{
COVERINGS OF INFINITE-DIMENSIONAL SPHERES
}

\author{
WILLIAM H. CUTLER
}

\begin{abstract}
Let $F$ be a normed linear space such that the countable infinite product of $F$ is homeomorphic to a normed linear space. (This is true for all Hilbert spaces, for example.) Let $S(F)$ denote the unit sphere in $F$. We prove the following

THEOREM 1. There is a countable cover of $S(F)$ of open sets each of which contains no pair of antipodal points.

THEOREM 2. There is a countable collection of closed sets in $S(F)$ the union of which contains exactly one member of each pair of antipodal points.

THEOREM 3. Let F be a Hilbert space. Then there is a countable collection of sets which cover $S(F)$ and whose diameters are less than 2.
\end{abstract}

Let $F$ be a normal linear space, and let $S(F)$ denote the unit sphere in $F$. We will prove several theorems in the case where $F^{\omega}$ (the countable infinite product of $F$ ) is homeomorphic to a normed linear space. Let us call this condition Property A. We note that Property A is satisfied by all finite-dimensional spaces (since $s$, the countable infinite product of lines, is homeomorphic to $l_{2}$, separable infinite-dimensional Hilbert space), and all Hilbert spaces (see [1] or [4]), and hence all spaces homeomorphic to a Hilbert space, such as reflexive Banach spaces [2]. The following theorems are proved for normed linear spaces with Property A.

THEOREM 1. There is a countable cover of open sets of $S(F)$ each of which contains no pair of antipodal points.

THEOREM 2. There is a countable collection of closed sets in $S(F)$ the union of which contains exactly one member of each pair of antipodal points.

THEOREM 3. Let $F$ be a Hilbert space. Then there is a countable collection of sets which cover $S(F)$ and whose diameters are less than 2 .

First, we note that Theorem 2 is proved by Klee for normed linear spaces of vector space dimension $\leqq c$ (the cardinality of the continuum) (Corollary 2.7 of [7]). We generalize this to spaces of all dimensions with the restriction that $F$ have Property A, and give a completely different proof. Theorem 3 is related to the following question.

Received by the editors June 27, 1972.

AMS (MOS) subject classifications (1970). Primary 57A20, 58B05.

Key words and phrases. Infinite-dimensional manifold, sphere, normed linear space, projective space.

(C) American Mathematical Society 1973 
Question. Let $F$ be Hilbert space of Schauder dimension c. Can $S(F)$ be covered by a countable collection of sets whose diameters are uniformly less than 2?

This question has important applications in graph theory discovered by Erdös. However, the methods of this paper do not suggest a solution to the question.

Restriction to the case $F \cong F^{\omega}$. We will now show that we need only prove the theorems for the case $F \cong F^{\omega}$. Since Theorems 2 and 3 are proved as corollaries to Theorem 1, we will only look at Theorem 1 . Let $G$ be the normed linear space homeomorphic to $F^{\omega}$. Look at the space $F \times G$ with norm $\|(x, y)\|=\|x\|_{F}+\|y\|_{G}$. Now $S(F)$ is naturally imbedded in $S(F \times G)$ as $S(F) \times\{0\}$, with antipodal points preserved, hence we need only prove Theorem 1 for $F \times G$. But $(F \times G)^{\omega} \cong\left(F \times F^{\omega}\right)^{\omega} \cong F^{\omega} \cong F \times F^{\omega} \cong F \times G$.

Therefore, throughout the rest of the paper, we will assume that $F \cong F^{\omega}$. (It is unknown whether this condition holds for all infinitedimensional Banach spaces.) We note that $F \cong l_{2} \times F$. This follows easily from the facts that $F \cong F^{\omega}$ and $F$ contains $R$ as a topological factor. Also, $S(F) \cong F($ see $[3])$.

Throughout the paper, $P(F)$ will denote $S(F)$ with antipodal points identified (projective $F$ space). We remark that the fundamental group of $P(F)$ is $Z / 2$ and all other homotopy groups are trivial. The projection map from $S(F)$ to $P(F)$ will be denoted by $\pi$.

\section{Several lemmas.}

LemMA 1. If $U$ is an open contractible set in $S(F)$ containing no pair of antipodal points, then $\pi(U)$ is contractible.

Proof. $\pi: U \rightarrow \pi(U)$ is a homeomorphism.

Lemma 2. There is a countable cover of $P\left(l_{2}\right)$ by contractible open sets.

Proof. Let $S\left(l_{2}\right)=\left\{\left(x_{1}, x_{2}, \cdots\right) \mid x_{i} \in R, \sum x_{i}^{2}=1\right\}$. Let

and

$$
U_{i}=\left\{\left(x_{1}, x_{2}, \cdots\right) \in S\left(l_{2}\right) \mid x_{i}>0\right\}
$$

$$
U_{i}^{\prime}=\left\{\left(x_{1}, x_{2}, \cdots\right) \in S\left(l_{2}\right) \mid x_{i}<0\right\} .
$$

Now let $V_{i}=\pi\left(U_{i}\right) \cdot \pi^{-1}\left(V_{i}\right)=U_{i} \cup U_{i}^{\prime}$ and the $U_{i}^{\prime}$ 's and $U_{i}^{\prime}$ 's cover $S\left(l_{2}\right)$. Hence the $V_{i}$ cover $P\left(l_{2}\right)$ and by Lemma 1 are contractible.

LEMMA 3. $P(F) \cong P\left(l_{2}\right) \times F$.

Proof. $P(F)$ has the same homotopy groups as $P\left(l_{2}\right) \times F$. Both are $F$-manifolds, and hence are ANR's. Hence they both have the homotopy 
type of a CW complex (p. 135 of [8]). The CW complexes are EilenbergMac Lane spaces (specifically $K(Z / 2,1) s)$, hence by p. 82 of [9] have the same homotopy type. But by [6], all homotopy-equivalent $F$-manifolds are homeomorphic.

Lemma 4. There is a countable cover of $P(F)$ by contractible open sets.

Proof. Let $V_{i}$ be the sets of Lemma 2 and look at $V_{i}^{\prime}=V_{i} \times F$. If $h: P(F) \rightarrow P\left(l_{2}\right) \times F$ is the homeomorphism of Lemma 3, then $h^{-1}\left(V_{i}^{\prime}\right)$ are the desired sets.

Lemma 5. Let $U$ be an open contractible set in $P(F)$. Then $\pi^{-1}(U)$ is the disjoint union of two open sets in $S(F)$ which are antipodals of each other.

Proof. Obviously, $\pi^{-1}(U)$ has either one or two components. If it has only one, then it is path-connected since we are in a manifold. Connect a pair of antipodal points with a path in $\pi^{-1}(U)$, say $L$. Then $\pi(L)$ is the image of a generator of the fundamental group of $P(F)$ which is $Z / 2$. Hence $U$ is not contractible.

Lemma 6. Let $F$ be a Hilbert space. There is a function $f:(0,2) \rightarrow(0,2)$ such that if $\varepsilon_{i} \rightarrow 0$ then $f\left(\varepsilon_{i}\right) \rightarrow 0$ satisfying: If $x, y \in S(F)$ and $d(x,-y)>$ $f(\varepsilon)$ then $d(x, y)<2-\varepsilon$.

Proof. Routine using trigonometry and the "roundedness" of Hilbert space spheres.

\section{Proof of the theorems.}

Proof of Theorem 1. Let $U_{i}$ be the cover of $P(F)$ by contractible open sets guaranteed by Lemma 4 . Using Lemma 5 , let $V_{i}, V_{i}^{\prime}$ be the components of $\pi^{-1}\left(U_{i}\right)$ and we are done.

Proof of Theorem 2. Let $V_{i}, V_{i}^{\prime}$ be as in the proof of Theorem 1. Write $V_{i}$ as the countable union of closed sets $C_{i, n}$. Let $C_{i, n}^{\prime}=C_{i, n}-$ $\bigcup_{j=1}^{i-1} V_{j}^{\prime}$. Then the $C_{i, n}^{\prime}$ are the desired sets.

Proof of Theorem 3. Let $V_{i}$ be the open sets in Theorem 1 for $F$ a Hilbert space. Let $V_{i, n}=\left\{x \in F \mid d\left(x, F-V_{i}\right)>f(1 / n)\right\}$ where $f$ is the function from Lemma 6 . Then the diameter of $V_{i, n}$ is $2-1 / n$ or less and $\bigcup_{n} V_{i, n}=V_{i}$.

We complete the paper with an alternative proof of the theorems for Hilbert spaces which avoids the high-powered results used in the proof of Lemma 3. We will prove Theorem 1 and the others follow as above.

Let $H(A)=\left\{f: A \rightarrow R \mid \sum_{a \in A}(f(a))^{2}<\infty\right\}$ where $f$ need not be continuous. ( $A$ is just an index set on the coordinates.) Since $A$ is infinite, let $B$ be a 
countably infinite subset of $A$. Let

$$
\begin{aligned}
& U_{b}=\{f: A \rightarrow R \mid f(b)>0\} \cap S(H(A)), \\
& U_{b}^{\prime}=\{f: A \rightarrow R \mid f(b)<0\} \cap S(H(A)) \text { for } b \in B .
\end{aligned}
$$

Let $K=\{f: A \rightarrow R|f| B=0\} \cap S(H(A))$. The sets $U_{b}, U_{b}^{\prime}$ are a countable collection of open sets whose union is $S(H(A))-K$.

Now we can regard $S(H(A))$ as $S(H(B)) * S(H(A-B))$ where $*$ denotes join. Since $S(H(B)) \cong l_{2}$ then $(S(H(A)), K)$ looks locally like $\left(H(A-B) \times l_{2}\right.$, $H(A-B) \times\{0\})$ and $K$ has local infinite deficiency in $S(H(A))$. Now we look at $P(H(A))$. It is an $H(A)$-manifold and $\pi(K)$ has local infinite deficiency. ( $K$ has local infinite deficiency in $X$ if every point of $K$ has an open neighborhood $U$ and a homeomorphism $h: U \rightarrow I_{2} \times Z$, for some $Z$, such that $h(U \cap K) \subset l_{2} \times$ pt. $)$ By the main theorem of [5], $\pi(K)$ is negligible, i.e. there is a homeomorphism $f: P(H(A))-\pi(K) \rightarrow P(H(A))$. Looking at the sets $\pi^{-1} f \pi\left(U_{b}\right)$ and applying Lemmas 1 and 5 , we are done.

\section{BIBLIOGRAPHY}

1. Cz. Bessaga, On topological classification of complete linear metric spaces, Fund. Math. 56 (1964/65), 251-288. MR 31 \#2580.

2. - Topological equivalence of unseparable reflexive Banach spaces. Ordinal resolutions of identity and monotone bases, Bull. Acad. Polon. Sci. Sér. Sci. Math. Astronom. Phys. 15 (1967), 397-399. MR 36 \#4321.

3. Cz. Bessaga and V. L. Klee, Two topological properties of topological linear spaces, Israel J. Math. 2 (1964), 211-220. MR 31 \#5055.

4. Cz. Bessaga and A. Pelczyński, Some remarks on homeomorphisms of Banach spaces, Bull. Acad. Polon. Sci. Sér. Sci. Math. Astronom. Phys. 8 (1960), 757-761. MR 24 \#A2230.

5. W. H. Cutler, Negligible subsets of infinite-dimensional Fréchet manifolds, Proc. Amer. Math. Soc. 23 (1969), 668-675. MR 40 \#2133.

6. D. W. Henderson and R. Schori, Topological classification of infinite-dimensional manifolds by homotopy type, Bull. Amer. Math. Soc. 76 (1970), 121-124. MR 40 \#4976.

7. V. L. Klee, Mappings into normed linear spaces, Fund. Math. 49 (1960/61), 25-34. MR 23 \#A3985.

8. A. P. Lundell and Stephen Weingram, The topology of $\mathrm{CW}$ complexes, Van Nostrand Reinhold, New York, 1969.

9. G. W. Whitehead, Homotopy theory, M.I.T. Press, Cambridge, Mass., 1966. MR 33 \#4935.

Department of Mathematics, Louisiana State University, Baton Rouge, LOUISIANA 70803 